\title{
ASTROMETRIC POSITIONS OF QUASARS \\ BY CCD OBSERVATIONS ${ }^{1}$
}

\author{
M. ASSAFIN \\ Observatório do Valongo, Univ. Federal do Rio de Janeiro \\ Rio de Janeiro, Brazil
}

AND

R. VIEIRA MARTINS, A.H. ANDREI

Observatório Nacional

Rio de Janeiro - RJ, Brazil

\begin{abstract}
We present results of CCD observations for 85 quasars with $-80^{\circ} \leq$ $\delta \leq+20^{\circ}$ declination, belonging to the International Celestial Reference System (ICRS). The positions are referred to the Carlsberg Series (CAMC) and PPM catalogues. We discuss a method which allows one to obtain precise positions based only on CCD observations, on the use of the Digitized Sky Survey of ST ScI and on the Guide Star Catalogue. The results are compared with the VLBI positions.
\end{abstract}

\section{Introduction}

Precise optical positions of ICRS quasars contribute to the link between the radio and fundamental reference frames. Orientation parameters of the ICRS relative to the FK5 system can then be derived. Due to the distinct magnitude ranges of extragalactic radiosources and of catalogue reference stars which represent the optical system, obtaining of quasar positions by the usual photographic techniques involves astrographic and long focus plate observations. To bypass the limiting precisions and large amount of work of such standard procedures, we devised a method that allows one to obtain quasar positions based only on CCD observations with a long focus telescope, on images extracted from the Digitized Sky Survey of STScI (DSS) and on the Guide Star Catalogue (GSC). An important step in the process is the correction of GSC star positions to the system of one of the astrometric catalogues, to use them as a secondary reference frame.

${ }^{1}$ Based on observations made at Laboratório Nacional de Astrofísica/CNPq/MCT

I. M. Wytrzyszczak, J. H. Lieske and R. A. Feldman (eds.), Dynamics and Astrometry of Natural and Artificial Celestial Bodies, 547, 1997.

(C) 1997 Kluwer Academic Publishers. Printed in the Netherlands. 


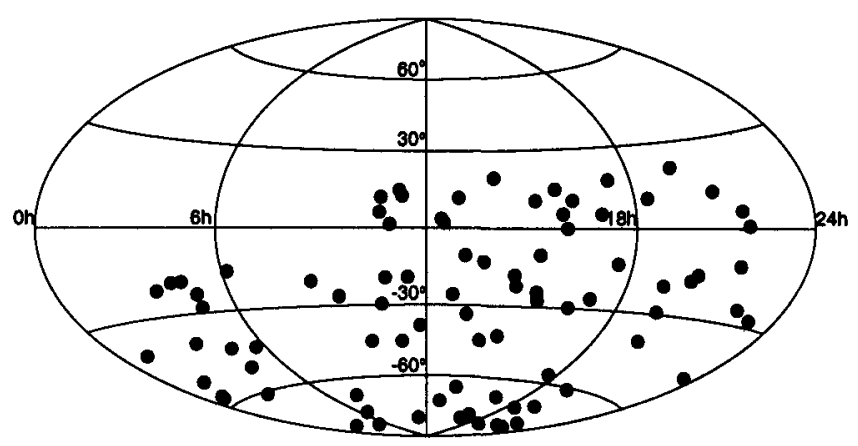

Figure 1. Sky distribution of the 85 programme quasars observed with CCD.

\section{The Reductions}

\subsection{OBSERVATIONAL PROGRAMME}

All 85 quasars belong to the new conventional extragalactic reference frame defined by the IAU - ICRS. The VLBI positions were taken from Ma et al. (1990), Sovers et al. (1988) and Russell et al. (1992). The range of brightness is $15 \leq$ magnitude $\leq 20$. The sky coverage is $-80^{\circ} \leq \delta \leq+20^{\circ}$.

The CCD observations were carried out at the $1.60-\mathrm{m}$ telescope of the Laboratório Nacional de Astrofísica, Itajubá-Brazil (plate scale $=13^{\prime \prime} / \mathrm{mm}$; $\lambda=+03^{h} 02^{m} 19$ s $\left.84 ; \phi=-22^{\circ} 23^{\prime} 54^{\prime \prime} .74\right)$. Four exposures were taken per source. The CCD/telescope resolution is 0 " $3 /$ pixel.

\subsection{POSITION REDUCTIONS}

All CCD and DSS objects were fitted by eliptical gaussians. Average $(x, y)$ errors were 0.060 for DSS and 0".015 for CCD images. The best DSS and $\operatorname{CCD}(\mathrm{x}, \mathrm{y})$ gaussian errors have given 0.030 and 0 ".005. There are no dependences with relative positions within the CCD or DSS fields. Magnitude dependences are similar to those found in Zacharias et al. (1995).

The PPM and CAMC reference catalogues were used to correct the GSC positions. The last one was a result of the unification of Carlsberg series 4 to 8 into a single catalogue (Puliaev and Andrei, 1996). The ACRS catalogue was also used. Therefore, 3 sets of quasar positions were obtained.

\subsection{GSC CORRECTION}

Considering its photographic nature, the original GSC star positions were corrected to the local system of astrometric catalogue stars using $(\xi, \eta)$ coor- 
TABLE 1. Standard deviations for the differences between the corrected or original GSC positions minus the reference catalogue positions, for the common stars located on $4^{\circ} \times 4^{\circ}$ sky regions around the source coordinates. The values refer to PPM and CAMC results.

\begin{tabular}{lcccc}
\hline Reference Catalogue & \multicolumn{2}{c}{ Cat - corrected GSC } & \multicolumn{2}{c}{ Cat - original GSC } \\
& $\sigma_{\alpha}$ & $\sigma_{\delta}$ & $\sigma_{\alpha}$ & $\sigma_{\delta}$ \\
\hline PPM & $0^{\prime \prime} 22$ & $\sigma^{\prime \prime} 19$ & $0^{\prime \prime} 46$ & $0^{\prime \prime} 29$ \\
CAMC & $0^{\prime \prime} 18$ & $0^{\prime \prime} 18$ & $0^{\prime \prime} 40$ & $0^{\prime \prime} 38$ \\
\hline
\end{tabular}

dinates in the tangential plane. The relationship between GSC and catalogue coordinates are given by classical $3^{\text {rd }}$ order polynomial models used in photographic astrometry. Table 1 displays standard deviations for the differences between the corrected or original GSC positions minus the reference catalogue positions, for the common stars. No dependences were found for the "corrected GSC positions minus reference catalogue star coordinates", with respect to magnitude or distance from the centre of projection (either quasar coordinates or even original GSC plate centres).

The 4 CCD fields per source are linked by $(x, y)$ adjustments ( 4 constants' model), following the Global Reduction Method (Benevides et al., 1992), adapted to our case. No dependences with distance from the CCD centre are found; magnitude dependences display the same features seen on the $(x, y)$ gaussian errors, with the same scale of values. For each quasar, the averaged CCD field obtained is then linked to a small DSS field through a $(x, y)$ adjustment ( 4 constants) between faint link ( $14 \leq$ magnitude $\leq$ 20 ) tertiary reference stars. The resulting field contains about 20 GSC stars plus the quasar, with the $(x, y)$ given in a common instrumental coordinate system. This field is then reduced with a $1^{\text {st }}$ order model with respect to the corrected GSC positions and the equatorial coordinates of the quasar are finally determined. The standard error of the CCD and DSS links were $00^{\prime \prime} 06$, which are consistent with the terciary $(x, y)$ gaussian errors of DSS images. The rms errors of the $(\alpha, \delta)$ reductions were also of about 0 ". 06 .

\section{Results}

The ACRS results have given larger standard deviations between optical and radio coordinates, and thus will not be considered. So, the fundamental system was represented by our optical radiosource positions derived from CAMC reductions and from PPM results for $\delta \leq-45^{\circ}$. We then used arclength differences to select fiducial sources, used to determine the orientation between the optical and radio frames. Results are shown in Table 2 for the 60 quasars thus selected. Rotation angles $\left(a_{1}, a_{2}, a_{3}\right)$ were derived following the procedures described in Arias et al. (1988). 
TABLE 2. Average "optical minus radio" differences, related standard deviations, and rotation angles for 60 quasars, selected by the use of arclenght differences.

\begin{tabular}{lrrr}
\hline & $\Delta \alpha \cos \delta$ & \multicolumn{1}{c}{$\Delta \delta$} \\
\hline average & $-0^{\prime \prime} 011$ & & -0.040 \\
standard deviation & $0^{\prime \prime} 134$ & & $0^{\prime \prime} 133$ \\
rotation angles & & $\left(-0^{\prime \prime} 035,-0^{\prime \prime} 024,+00^{\prime \prime} 025\right)$ & \\
and errors & & $\left(0^{\prime \prime} 021,0.0^{\prime \prime} 022, \quad 0^{\prime \prime} 022\right)$ & \\
\hline
\end{tabular}

\section{Conclusions}

The comparisons between our results and the radio frame are consistent with the erros of the optical reference catalogues used. The radio and optical reference frames agreed within 0 "'15 (standard deviation) with less than 0 ."04 average positional offsets. Both systems appear to be well aligned within 0 !'04, displaying orientation angles of $\left(a_{1}=-0.035, a_{2}=-0{ }^{\prime \prime} 024\right.$, $\left.a_{3}=+0 . \prime 025\right)$.

The results support the GSC correction method developed and the use of CCD in combination with the DSS, for high precision optical astrometry of quasars. The future use of the HIPPARCOS or TYCHO catalogues in the GSC correction method will enable to extend the spacial frame up to the $15^{\text {th }}$ magnitude, thus allowing the achievement of more precise optical positions of quasars that may contribute to improve the alignment between the HIPPARCOS and ICRS frames.

It is noteworthy that the procedures are very simple and useful for small field astrometry of natural satellites, asteroids and faint objects in general, as well as for the determination of precise positions of objects scheduled for fiber optics spectroscopy.

Acknowledgements. We are thankful to the LNA/MCT for the allocated nights and staff assistence at the $1.60-\mathrm{m}$ telescope. This work was supported by Observatório Nacional/CNPq. The Digitized Sky Survey was produced at the Space Telescope Science Institute.

\section{References}

Arias, E.F., Feissel, M., Lestrade, J.-F.: 1988, Astron. Astrophys. 199, 357.

Benevides-Soares, P., Teixeira, R.: 1992, Astron. Astrophys. 253, 307.

Ma, C., et al.: 1990, Astron. J. 99, 1284.

Puliaev, S. and Andrei, A.H.: 1996, this volume.

Russel, J.L., et al.: 1992, Astron. J. 103, 2090.

Sovers, O.J., Edwards, C.D., Jacobs, C.S., Lanyi, G.E., Liewer, K.M., and Treuhaft, R.N.: 1988, Astron. J. 95, 1647.

Zacharias, N., de Vegt, Ch., Winter, L., and Johnston, K. J.: 1995, Astron. J. 110, 3093. 